8. Crisis Management Department, Tokushima Zero Initiative Promotion Division, Tokushima Prefecture, Tokushima city, Japan

Introduction: Nankai Trough earthquake, with an anticipated death toll of 323,000, is a disaster for which the country of Japan set the highest priority on building capacities. Tokushima prefecture aims to minimize preventable death among survivors and has strived to build a medical and health response system and strengthen outreach systems for vulnerable populations. To actualize these aims, Tokushima prioritized human resource development.

Methods: Tokushima has initiated periodic trainings based on the Sphere Standard, the internationally recognized minimum standards for humanitarian aid, since 2015. The trainings were conducted by certified trainers and trainees received an official certification recognized by the Sphere Project, Geneva. The training materials were localized and the trainings were contextualized to Japan as a developed and super-aged nation. The learning outcome was evaluated by a pre-post test.

Results: Between April 2015 and November 2018 the two-day training was held seven times. There were two hundred twelve participants from various clusters such as health, education, logistics, nutrition and food, security, and protection. The results of the pre-post test were statistically significant (still in process) indicating the effectiveness of the training on knowledge. Training evaluations suggest nurturing ethical attitudes and skills utilizing the Sphere Handbook.

Discussion: Despite under-recognizing the Sphere Standard in Japan, the Standard has been incorporated into the disaster risk reduction plan in Tokushima. For larger scale human resource development, training local representatives to be trainers would be the next step.

Prehosp Disaster Med 2019;34(Suppl. 1):s152-s153

doi:10.1017/S1049023X19003431

Novel Delivery of Meaningful EMS and Disaster Medicine Content to Residents and Medical Students

Dr. Lindsay Flax ${ }^{1}$, Dr. E. Liang Liu ${ }^{1}$, Dr. Brian Miller ${ }^{1}$, Dr. Brandon Morshedi ${ }^{1}$, Dr. Raymond L. Fowler ${ }^{1}$, Dr. Raymond E. Swienton ${ }^{1}$, Dr. Josh Mugele ${ }^{2}$, Dr. Kelly Klein ${ }^{1}$

1. Department of Emergency Medicine, University of Texas Southwestern Medical Center, Dallas, United States

2. Indiana University School of Medicine, Indianapolis, United States

Introduction: Residency education delivery in the United States has migrated from conventional lectures to alternative educational models that include mini-lectures, small group, and learner lead discussions. As training programs struggle with mandated hours of content, prehospital (EMS) and disaster medicine are given limited focus. While the need for prehospital and disaster medicine education in emergency training is understood, no standard curriculum delivery has been proposed and little research has been done to evaluate the effectiveness of any particular model.

Aim: To demonstrate a four-hour multi-modal curriculum that includes lecture based discussions and small group exercises, culminating in an interactive multidisciplinary competition that integrates the previously taught information.

Methods: EMS and disaster faculty were surveyed on the previous disaster and prehospital educational day experiences to evaluate course content, level of engagement, and participation by faculty. Based on this feedback, the EMS/Disaster divisions developed a schedule for the four hour EMS and Disaster Day that incorporated vital concepts while addressing the pitfalls previously identified. Sessions included traditional lectures, question and answer sessions, small group exercises, and a tabletop competition. Structured similarly to a strategy board game, the tabletop exercise challenged residents to take into account both medical and ethical considerations during a traditional triage exercise.

Results: Compared to past reviews by emergency medical faculty, residents, and medical students, there was a precipitous increase in satisfaction scores on the part of all participants.

Discussion: This curriculum deviates from the conventional education model and has been successfully implemented at our 3-year residency program of 66 residents. This EMS and Disaster Day promotes active learning, resident and faculty participation, and retention of important concepts while also fostering relationships between disaster managers and the Department of Emergency Medicine.

Prehosp Disaster Med 2019;34(Suppl. 1):s153

doi:10.1017/S1049023X19003443

\section{A Novel Strategy to MCI Management}

Dr. Yun-Kuan Lin, Dr. Pei-Fang Lai, Dr. Kuang-Yu Niu

Hualien Tzu Chi Hospital, Hualien, Taiwan

Introduction: On February 6, 2018, a 6.0 magnitude earthquake struck Hualien, a county of East Taiwan. Hualien Tzu Chi Hospital, the only tertiary hospital in East Taiwan, activated the mass casualty incident (MCI) call and received 144 patients that night. Our operation did not perform satisfactorily despite regular MCI drills. Thus, a new strategy to cope with the increasing frequency of disaster-related MCIs was developed.

Aim: To facilitate the management of disaster-related MCIs, we developed a novel Disaster Response System which includes a triage system combining Simple Triage and Rapid Treatment (START) and Five-Level Taiwan Triage and Acuity Scale (TTAS), a novel registration system for MCIs, and anonymous patient identification and reporting system.

Methods: We begin the triage with the START method and then shift to the TTAS. The new registration system only needs the patient's gender, age, and triage category. Patients are then assigned to different treatment areas accordingly. Further dispositions are applied after initial stabilization management. To identify the anonymous disaster victims, we take photographs of victims after clean-up and display them on an electronic bulletin with the patient list to the families in our emergency department. Real-time casualty statistics are collected automatically and synchronized to the governmental administrative system.

Results: This novel Disaster Response System reduces the time from patient arrival to definite treatment and disposition in a simulated mass casualty incident exercise. The victim 